\title{
Processos hidrológicos em uma sub-bacia do Parque Estadual da Serra do Mar, núcleo Santa Virgínia
}

\author{
Hydrological processes in wathershed within the State Park of Serra do Mar, \\ Santa Virgínia stewardship
}

\author{
Juliano Daniel Groppo ${ }^{\mathrm{I}}$, Luiz Felippe Salemi' ${ }^{\mathrm{II}}$, Jorge Marcos Moraes ${ }^{\mathrm{III}}$, \\ Rodrigo Trevisan $^{\mathrm{IV}}$, Luiz Antônio Martinelliv
}

\begin{abstract}
Resumo
O presente estudo busca caracterizar o funcionamento hidrológico de uma sub-bacia com cobertura de Mata Atlântica, localizada no Parque Estadual da Serra do Mar, núcleo Santa Virgínia. Para isso, foram monitorados entre os anos de 2008 e 2009, alguns processos hidrológicos: escoamento superficial, umidade do solo $(0,15,0,30,0,50$ e 0,90 metros de profundidade), transprecipitação, precipitação e vazão, através de nove parcelas de escoamento superficial, tensiômetros, pluviômetros comuns, pluviógrafo tipo báscula e calha tipo $\mathrm{H}$, respectivamente. Com o intuito de elucidar os processos hidrológicos que geram escoamento direto, mediu-se a condutividade hidráulica do solo $\left(\mathrm{K}_{\mathrm{sat}}\right)$ nas mesmas profundidades da umidade do solo, com a utilização de um permeâmetro de carga constante. Os resultados evidenciaram que em 2008, os valores de precipitação (P), interceptação (I), transpiração (T) e deflúvio (Q) foram de $1716 \mathrm{~mm}, 574 \mathrm{~mm}$ (33\%), $461 \mathrm{~mm}$ (27\%) e $681 \mathrm{~mm}(40 \%)$, ao passo que, em 2009, esses valores foram de, respectivamente, $3003 \mathrm{~mm}, 960 \mathrm{~mm}(32 \%), 610 \mathrm{~mm}(20,3 \%)$ e $1433 \mathrm{~mm}(48 \%)$. O predomínio de intensidades de chuva de 0 a $5 \mathrm{~mm} \cdot \mathrm{h}^{-1}$ e geralmente valores superiores de $\mathrm{K}_{\text {sat }}$ a $0,15 \mathrm{~m}$ de profundidade faz com que quase sua totalidade da se infiltre no solo. Como consequência, há uma baixa geração de escoamento superficial e os potenciais matriciais da água do solo próximos a zero são observados ao longo do ano. A maior precipitação apresentada em 2009 em relação a 2008 causou aumento significativo na precipitação interna, escoamento superficial e deflúvio diário elucidando a variabilidade temporal que anos mais úmídos podem provocar na dinâmica hidrológica de bacias cobertas por Mata Atlântica.
\end{abstract}

Palavras-chave: Hidrologia florestal; Monitoramento; Mata Atlântica

\begin{abstract}
The present paper aimed to describe the hydrological functioning of a small catchment covered by the Atlantic rainforest within the State Park of Serra do Mar, Sao Paulo state, Brazil. To reach these goals, the hydrological processes were monitored during 2008 and 2009. The surface runoff, groundwater, streamflow, precipitation, net precipitation and soil water storage at $0.15 \mathrm{~m}, 0.30 \mathrm{~m}, 0.50 \mathrm{~m}$ and $0.90 \mathrm{~m}$, were monitored through nine $2.25 \mathrm{~m} 2$ plots, tensiometer, tipping-bucket rain gauges, trough-type collectors and $\mathrm{H}$-flume, respectively. Aiming to clarify the soil hydrological processes, the soil saturated hydraulic conductivity was measured in the field at the depths of water storage measurements using a constant head permeameter. For 2008, the annual precipitation (P) was 1716 $\mathrm{mm}$. Interception losses accounted for $33 \%$ of the annual precipitation. The runoff coefficient was $40 \%$ of annual rainfall. For 2009, annual precipitation was $3003 \mathrm{~mm}$. Approximately $32 \%$ of rainfall was intercepted. Runoff coefficient was $48 \%$ of annual rainfall. The predominance of low rainfall intensity $\left(0-5 \mathrm{~mm} \mathrm{~h}^{-1}\right)$ substantially below the $\mathrm{K}_{\text {sat }}$ median at $0.15 \mathrm{~m}$ allowed us to infer that the soil accommodates the majority of the rain events. This inference is corroborated by low surface runoff coefficients. The higher precipitation in 2009 caused a significant increase in throughfall, surface runoff, a daily flow relative to 2008, showing the temporal variability that high precipitation years promote on the hydrologic dynamic of catchments covered by the Atlantic Rainforest.
\end{abstract}

Keywords: Forest hydrology; Monitoring; Atlantic Forest

I Engenheiro Ambiental, Dr., Professor da Universidade Vale do Rio Doce, Rua Israel Pinheiro, 2000, Bairro Universitário, CEP 35020-220, Governador Valadares (MG), Brasil. jdgroppo@gmail.com (ORCID: 0000-0002-4820-1632)

II Gestor Ambiental, Dr., Professor da Universidade de Brasília, Ciências da Vida e da Terra, Campus Planaltina, Área Universitária, 01, Bairro Vila Nossa Senhora de Fátima, CEP 73345-010, Planaltina (DF), Brasil. piposalemi@gmail.com (ORCID: 0000-0003-2271-5712)

III Engenheiro Mecânico, Dr., Professor da Escola de Engenharia de Piracicaba, Av. Monsenhor Martinho Salgot, 560 Bairro Areião, CEP 13414-040, Piracicaba (SP), Brasil. jmmoraes066@gmail.com (ORCID: 0000-0001-7945-2381)

IV Engenheiro Ambiental, MSc., Pesquisador Autônomo, Rua das Begônias, 190, bairro Nova Piracicaba, CEP 13405-072, Piracicaba (SP), Brasil. digaotrev@yahoo.com.br (ORCID: 0000-0002-7211-4459)

V Engenheiro Agrônomo, Dr., Universidade de São Paulo, Centro de Energia Nuclear na Agricultura, Av. Centenário, 303, Bairro São Dimas, CEP 13400-970, Piracicaba (SP), Brasil. zebu@cena.usp.br (ORCID: 0000-0002-7103-7551) 


\section{Introdução}

A Mata Atlântica que originalmente ocupava quase toda a costa brasileira, com cerca de 1,3 milhões $\mathrm{km}^{2}$, assim como a maioria dos biomas florestais tropicais, sofreu intenso desmatamento. Atualmente, este sistema natural ocupa área descontínua de aproximadamente $98.800 \mathrm{~km}^{2}$, isto é, 7,6\% da sua extensão original (FUNDAÇÃO SOS MATA ATLÂNTICA, 2006; RIBEIRO et al., 2009). Essas áreas remanescentes estão distribuídas em fragmentos de diversos tamanhos, formas, estágios de sucessão e situação de conservação (LIRA et al., 2012). As manchas disjuntas da floresta existem particularmente em locais de topografia muito acidentada, que impede qualquer atividade agrícola (LEITÃO-FILHO, 1987), ou estão protegidas na forma de unidades de conservação tais como parques estaduais e federais, reservas biológicas e estações ecológicas.

Apesar de reduzida a poucos fragmentos de valor substancial para a conservação da diversidade biológica, a Mata Atlântica ainda é um dos biomas que apresenta maior biodiversidade no mundo (MURRAY-SMITH et al., 2009). Devido à grande degradação e importância, a Mata Atlântica é considerada por entidades nacionais e internacionais como um dos biomas prioritários para execução de políticas de conservação (hotspot) em função do alto grau de endemismo de suas espécies (MYERS et al., 2000; ROCHA et al., 2004).

Dentre os atributos principais das florestas podem-se citar a função hidrológica, por desempenhar papel fundamental na captação e distribuição de água de chuva, no suprimento de água para recarga dos aquíferos, assim como na regulação do escoamento superficial (ARCOVA; CICCO, 1997). A conversão de áreas florestais tem como consequência, alterações significativas nos processos hidrológicos, tais como: formação de camadas impedimento na superfície do solo ou próximo a ela de forma a favorecer a ocorrência de escoamento superficial ou subsuperficial (ELSENBEER, 2001; GERMER et al., 2010); diminuição da intercepção e da evapotranspiração (WRIGHT et al., 1996); aumento dos níveis de umidade do solo (HODNETT et al., 1995; MORAES et al., 2006) e de recarga de águas subterrâneas (JIPP et al., 1998); diminuição da capacidade de infiltração e de armazenamento de água do solo.

A maioria dos estudos que vem sendo realizado na Mata Atlântica estão focados na fauna e na flora (MURRAY-SMITH et al., 2009). Considerando a importância desse bioma, poucas informações estão disponíveis em relação ao seu funcionamento, especialmente com relação aos aspectos hidrológicos. Nesse sentido, entender como a variação da pluviosidade altera o funcionamento hidrológico de sub-bacias cobertas por estas florestas se torna igualmente relevante em termos de compreensão da dinâmica hidroecológica desses sistemas, analisar a dinâmica da sub-bacia em situações de diferentes pluviosidades anuais.

Dentro desse contexto, o presente estudo teve como objetivo caracterizar o funcionamento hidrológico de uma sub-bacia inteiramente coberta por Mata Atlântica (Floresta Ombrófila Densa) localizada no Parque Estadual da Serra do Mar, núcleo de Santa Virgínia, entre os anos 2008 e 2009.

\section{Material e métodos}

\section{Área de estudo}

A sub-bacia de estudo possui uma área de 11,5 hectares (ha) e $28 \pm 14 \%$ de declividade média, localizada na latitude de $23^{\circ} 19^{\prime} 19^{\prime}$ 'S e $45^{\circ} 05^{\prime} 56^{\prime \prime}$ W de longitude, está localizada no Parque Estadual da Serra do Mar (PESM), Núcleo Santa Virginia (Figura 1). A referida unidade de conservação representa a maior porção contínua preservada de Mata Atlântica do Estado de São Paulo. 


\section{Figura 1 - Área de estudo.}

Figure - 1 Study area.

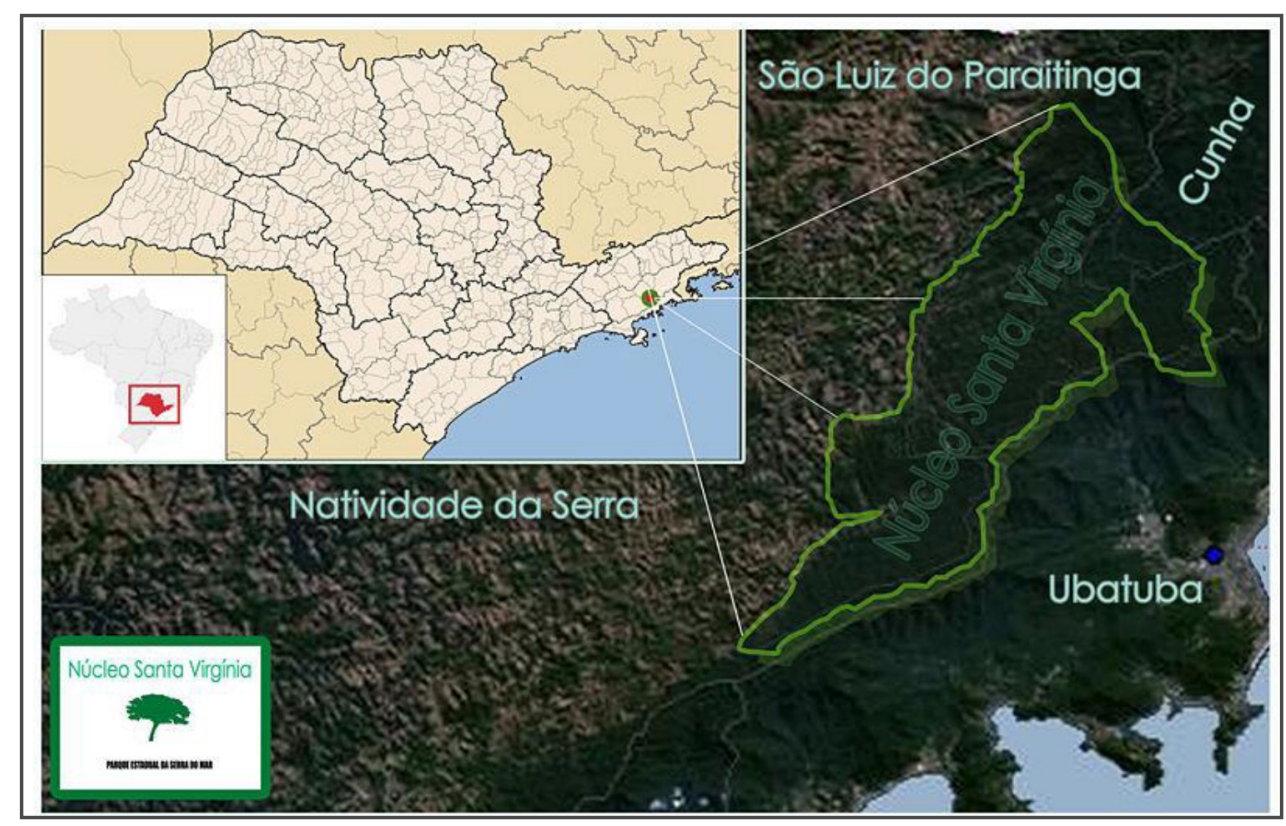

Fonte: São Paulo (2006)

O Núcleo Santa Virgínia é um dos núcleos de gerenciamento do Parque Estadual da Serra do Mar e abrange parte dos municípios de São Luís do Paraitinga (70\%), Cunha (20\%) e Ubatuba (10\%), e possui aproximadamente 5000 ha de extensão. Tal núcleo é coberto predominantemente por Floresta Primária, Floresta Ombrófila Densa Montana (VELOSO; RANGEL FILHO; LIMA, 1991). O núcleo situa-se a uma altitude que varia de $850 \mathrm{~m}$ a $1.100 \mathrm{~m}$. O clima regional é tropical temperado, sem estação seca bem definida, com precipitação média anual superior a $2.000 \mathrm{~mm}$. A temperatura média anual varia de $21^{\circ} \mathrm{C}$ (de $19^{\circ} \mathrm{C}$ no inverno a $25^{\circ} \mathrm{C}$ no verão) na costa até $21^{\circ} \mathrm{C}$ no planalto (TABARELLI; MANTOVANI, 1999).

Os solos da microbacia são predominantemente caracterizados pela presença de horizonte B incipiente (Cambissolos), com horizontes: A $(0-0,25 \mathrm{~m}), \mathrm{B}(0,25-1,10 \mathrm{~m})$ e C $(1,10-+1,30 \mathrm{~m})$ de textura média. Nas partes mais baixas da bacia há a ocorrência de solos aluviais (Neossolos Flúvicos e Cambissolos Flúvicos).

\section{Delineamento experimental}

O monitoramento da precipitação, precipitação interna, escoamento superficial, potencial matricial da água do solo e vazão foi realizado semanalmente de janeiro a dezembro de 2008 e quinzenalmente de janeiro a dezembro de 2009. O delineamento experimental é ilustrado na Figura 2.

A precipitação foi medida através de um pluviógrafo tipo de báscula, com resolução 0,254 $\mathrm{mm}$, instalado em uma clareira situada a aproximadamente de $1 \mathrm{~km}$ fora da microbacia de estudo. Tal equipamento registrou a precipitação em intervalos de tempo de 5 minutos. A precipitação que atravessa o dossel da floresta (precipitação interna) foi medida utilizando 10 coletores de PVC com $2 \mathrm{~m}$ de comprimento com abertura transversal de $0,15 \mathrm{~m}^{2}$ de área, ligados a um galão. Informações mais detalhadas estão disponíveis em Groppo (2010). 
Figura 2 - Diagrama esquemático do delineamento do experimento na sub-bacia de estudo.

Figure 2 - Schematic diagram of the experimental setup installed in the catchment.

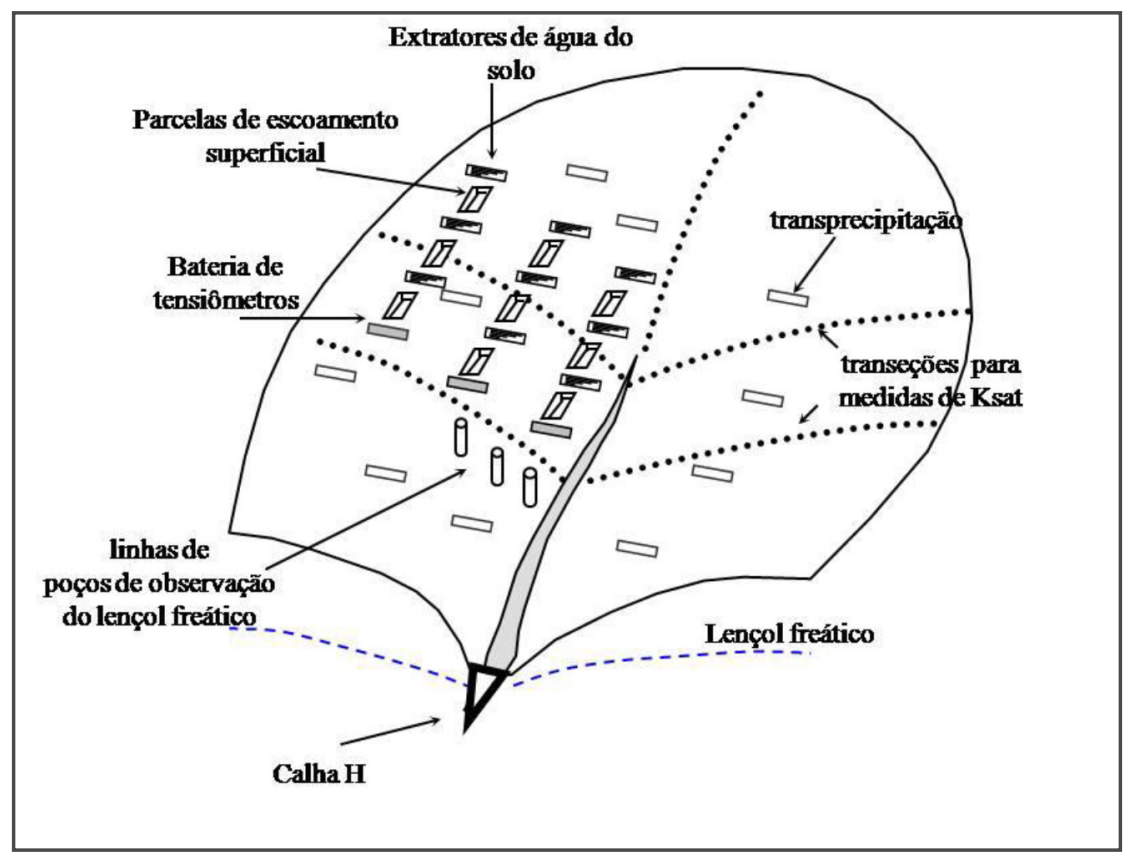

Fonte: Autores (2019)

O escoamento superficial foi medido através de nove parcelas com 1,5 m x 1,5 m de dimensão, em triplicata e em três pontos da vertente (superior, médio e inferior). O ponto superior foi instalado próximo ao interflúvio, o inferior próximo ao canal fluvial, porém fora da área de preservação permanente, e o ponto médio entre esses dois últimos.

O potencial matricial da água do solo foi medido através de três baterias de tensiômetros comuns, nas profundidades de $0,15,0,30,0,50$ e $0,90 \mathrm{~m}$, nos mesmos três pontos da vertente utilizados para as parcelas de escoamento superficial, descritos anteriormente. As medidas de potencial matricial da água do solo foram realizadas com o auxílio de um manômetro digital.

A vazão no canal foi medida utilizando-se uma calha do tipo $\mathrm{H}$ situado no exutório da sub-bacia acoplada a um sensor eletrônico de nível de água integrado, de resolução de $1 \mathrm{~mm}$, que registrou a altura da lâmina a cada 5 minutos. Deste modo, a descarga foi calculada aplicando a altura da água medida em metros na equação:

$$
\log \mathrm{Q}=0,0228+2,5473 * \log \mathrm{H}+0,2540 *(\log H)^{2}
$$

Assim, a vazão era obtida em $1 . \mathrm{s}^{-1}$, e posteriormente transformada em deflúvio $(\mathrm{mm})$ utilizando área de estudo.

Com a combinação hietograma-hidrograma, foi possível entender a geração de escoamento direto. A separação do escoamento superficial direto foi realizada segundo metodologia proposta por Chow, Maidment e Mays (1988) e Tucci (2001), em que, durante o evento pluvial, o comportamento do escoamento de base é considerado linear.

Considerando-se: 1) a variação do armazenamento de água anual no solo como desprezível (FILL, 1987) como é o caso dessa região em que não há estação seca definida; 2) vazamentos pelo 
substrato rochoso ausentes; e 3) ausência de fluxos laterais para fora ou para dentro da sub-bacia, o balanço hídrico foi calculado pela seguinte equação:

$$
\mathrm{P}=\mathrm{Q}+\mathrm{ET}
$$

Em que: $P$ é a precipitação $(\mathrm{mm}) ; \mathrm{Q}$ é o deflúvio; e $E T$ é a evapotranspiração.

Para o cálculo da estimativa da transpiração foi utilizada a seguinte equação: sendo,

$$
\begin{aligned}
& \mathrm{ET}=\mathrm{I}+\mathrm{T} \\
& \mathrm{T}=\mathrm{P}-(\mathrm{Q}+\mathrm{I})
\end{aligned}
$$

Em que: I é a interceptação ( $\mathrm{mm})$; e $T$ é a transpiração $(\mathrm{mm})$.

No presente estudo, assumiu-se que a precipitação efetiva equivale a precipitação interna dado que a o escoamento pelo tronco pode ser considerado inferior a $1 \%$ em florestas de estrutura similar (ARCOVA; CICCO; ROCHA, 2003).

Medidas da condutividade hidráulica do solo na saturação $\left(\mathrm{K}_{\mathrm{sat}}\right)$ foram realizadas em 25 pontos distribuídos em transectos lineares das margens do canal fluvial ao interflúvio (Figura 2), empregando-se um permeâmetro compacto de carga constante (AMOOZEGAR, 1992) em quatro profundidades do solo $(0,03-0,15 \mathrm{~m} ; 0,12-0,30 \mathrm{~m} ; 0,32-0,50 \mathrm{~m} ; 0,72-0,90 \mathrm{~m})$.

\section{Análises Estatísticas}

O teste não paramétrico Kruskal-Wallis foi empregado para detectar diferenças entre as quatro profundidades de $\mathrm{K}_{\text {sat }}$. Em caso de detecção de diferenças, aplicou-se o teste MannWhitney para encontrar quais profundidades diferem entre si. Este último teste também foi empregado para detectar diferenças com relação à precipitação interna, o potencial matricial do solo, o escoamento superficial e o deflúvio entre os dois anos de estudos (2008 e 2009).

Todas as análises foram realizadas no pacote estatístico Minitab (versão 16). Diferenças menores que $\mathrm{p}<0,05$ foram consideradas significativas.

\section{Resultados}

A precipitação apresentou uma diferença percentual de $60 \%$ entre os dois anos de estudo, sendo $1.716 \mathrm{~mm}$ no ano de 2008 e $3.003 \mathrm{~mm}$ em 2009 (Figura 3). A estação menos úmida ocorreu entre os meses de maio e julho de 2008 , com aproximadamente $8 \%$ do total precipitado. Nesse ano, o mês de julho foi o menos chuvoso de todo o período estudado com apenas $4 \mathrm{~mm}$. No ano de 2009, todos os meses apresentaram precipitação mais elevada em relação ao anterior, sendo a mínima observada no mês de maio $(74 \mathrm{~mm})$ e a máxima no mês de dezembro $(748 \mathrm{~mm})$. Esse último valor representou aproximadamente $25 \%$ do total precipitado do ano de 2009 .

Em relação à intensidade de chuva, observou-se que, ao considerar ambos os anos, $84 \%$ dos eventos estão na faixa de 0 a $4,9 \mathrm{~mm} \mathrm{~h}^{-1}$, e que a contribuição desta classe de intensidade no volume total de chuva foi de $54 \%$ no ano de 2008 e de $36 \%$ no ano de 2009 (Figura 4). Ao se considerar a faixa de 0 a $9,9 \mathrm{~mm} \cdot \mathrm{h}^{-1}$, a contribuição do total precipitado aumenta para em torno de $70 \%$ no ano de 2008 e para em torno de $50 \%$ em 2009. Já os eventos com intensidades mais altas ocorrem com menor frequência, se comparados aos de baixa intensidade. Por outro lado, eventos com intensidades superiores a $30 \mathrm{~mm} \cdot \mathrm{h}^{-1}$, apesar de pouco frequentes, contribuíram com aproximadamente $12 \%$ do total precipitado no ano de 2008 e $22 \%$ no ano de 2009 . 
Figura 3 - Distribuição mensal da precipitação.

Figure 3 - Monthly distribution of precipitation.

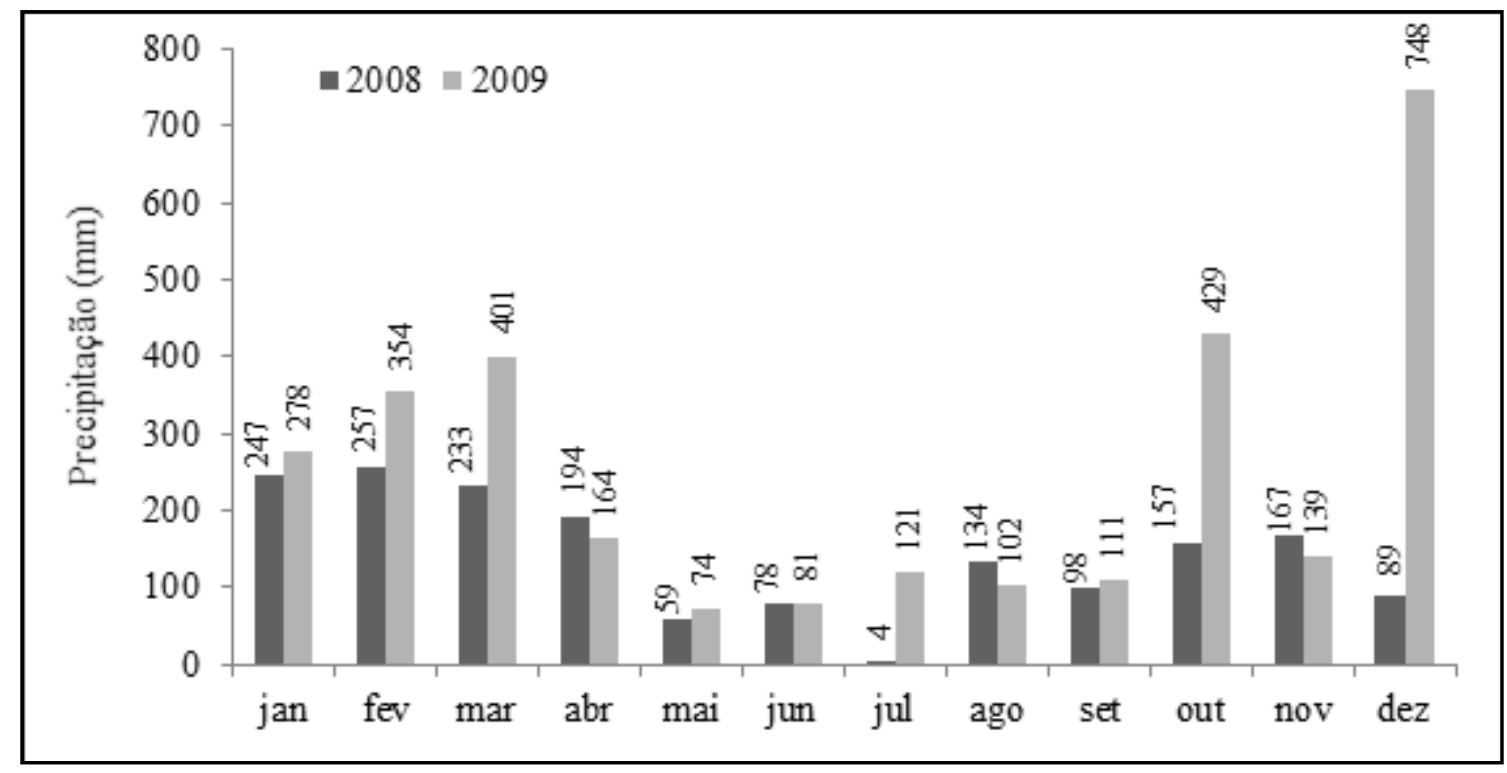

Fonte: Autores (2019)

\section{Figura 4 - Distribuição de frequência e contribuição de cada classe de intensidade de precipitação.}

Figure 4 - Frequency distribution and contribution of each class of precipitation intensity.

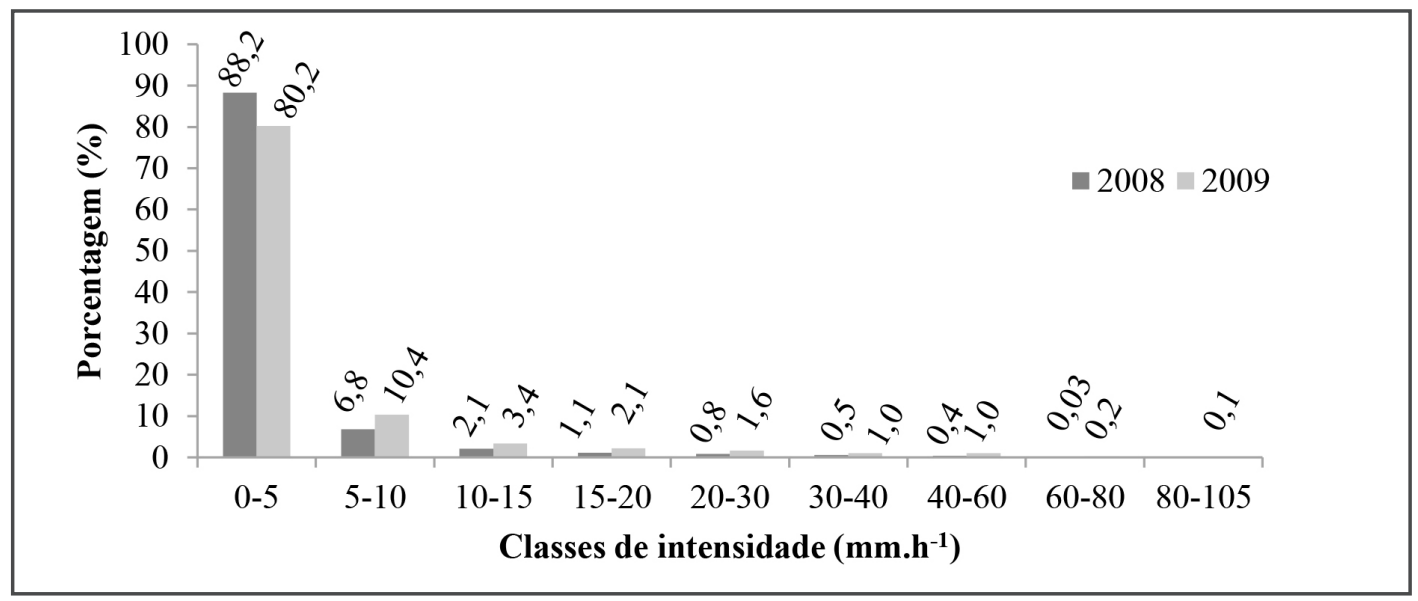

Fonte: Autores (2019)

No que se refere à precipitação interna, apesar da maior quantidade de chuva no ano de 2009 e maior contribuição de chuvas mais intensas, a relação entre a precipitação e interceptação foi similar em termos percentuais, com 33\% (574 mm) em 2008 e 32\% (960 mm) em 2009. Além disso, através do teste de Mann-Whitney pode-se observar diferença significativa na precipitação interna entre os dois anos estudados.

Os valores da condutividade hidráulica do solo na saturação $\left(\mathrm{K}_{\mathrm{sat}}\right)$ apresentaram grande variabilidade entre os pontos em todas as profundidades. Houve a tendência da mediana e da variabilidade decrescer com o aumento da profundidade (Figura 5). Os valores da mediana 
variaram de $61 \mathrm{~mm} \cdot \mathrm{h}^{-1}$ próximo à superfície $(0,03-0,15 \mathrm{~m})$, para $11 \mathrm{~mm} \cdot \mathrm{h}^{-1}, 2 \mathrm{~mm} \cdot \mathrm{h}^{-1}$ e $5 \mathrm{~mm} \cdot \mathrm{h}^{-1}$ nas profundidades de $0,15,0,30,0,50$ e $0,90 \mathrm{~m}$, respectivamente. As seguintes diferenças significativas de $\mathrm{K}_{\mathrm{sat}}$ foram detectadas entre as diferentes profundidades do solo: $[0,15 \mathrm{~m}]>[0,30 \mathrm{~m}]>[0,50 \mathrm{~m} \sim$ $0,90 \mathrm{~m}]$. O sinal “ $~$ ” foi empregado para mostrar que não existem diferenças significativas entre as duas profundidades $(a=0,05)$.

Figura 5 - Gráfico Box-Plot dos valores da $K_{\text {sat }}$ nas profundidades de 0,15 m, 0,30 m, 0,50 m e $0,90 \mathrm{~m}$ ( $\mathrm{n}=25$ para cada profundidade). A linha horizontal dentro das caixas representa a mediana, as linhas horizontais das caixas representam o primeiro e o terceiro quartil e as barras verticais os valores máximos e mínimos. A linha pontilhada na horizontal representa a intensidade de chuva de $5 \mathrm{~mm} \cdot \mathrm{h}^{-1}$ que é a mais representativa da região de estudo.

Figure 5 - Box-Plot of the $\mathrm{K}_{\text {sat }}$ values at the depths of $0.15 \mathrm{~m}, 0.30 \mathrm{~m}, 0.50 \mathrm{~m}$ and $0.90 \mathrm{~m}(\mathrm{n}=$ 25 for each depth). A horizontal line inside the boxes represents a median, as horizontal lines of the boxes represent the first and third quartiles and as vertical bars the maximum and minimum values. The horizontal dotted line represents a rainfall intensity of $5 \mathrm{~mm} . \mathrm{h}-1$, which is most representative of the study region.

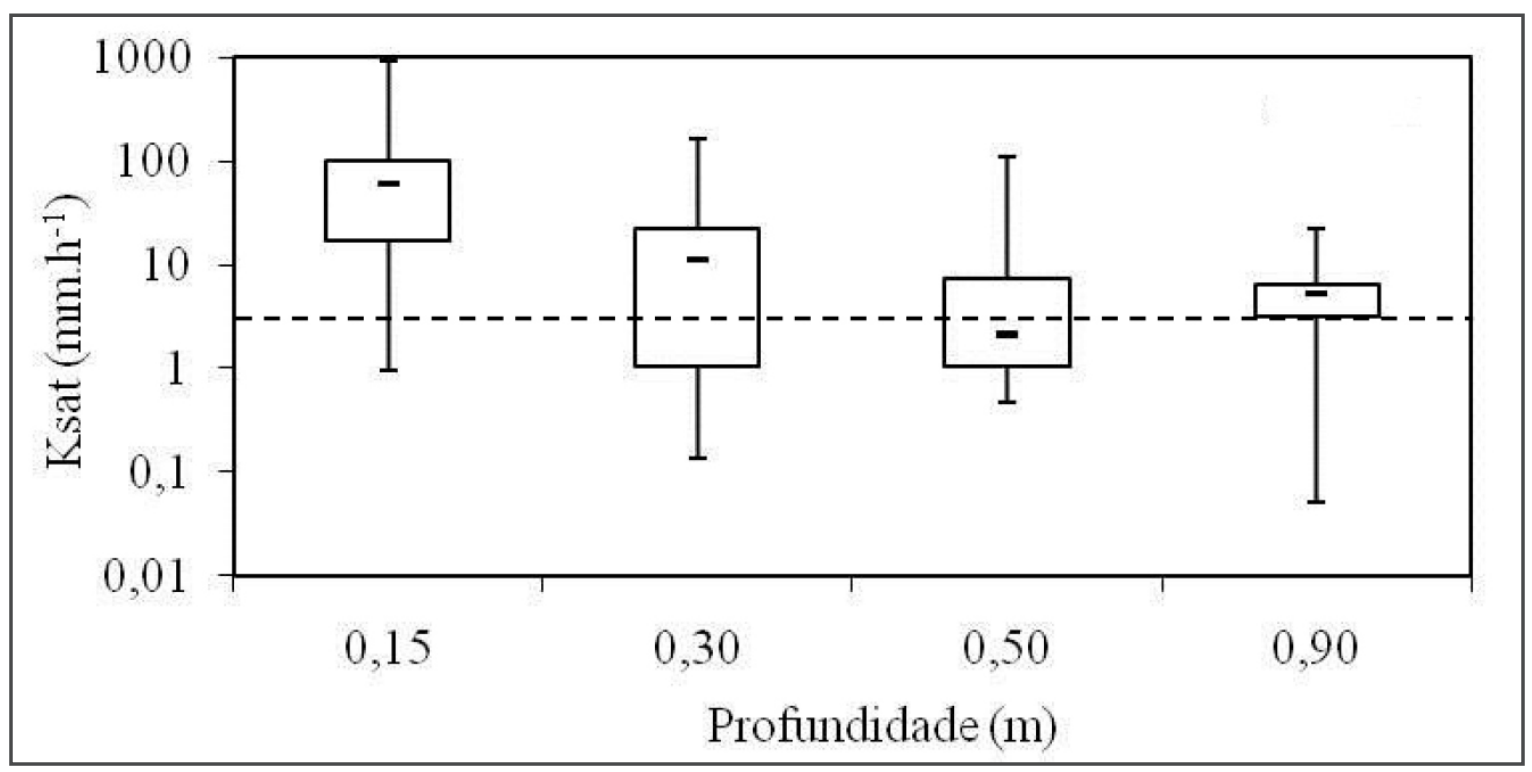

Fonte: Autores (2019)

Por meio das medições realizadas nos poços a 0,5 metros de profundidade não foi observado lençol suspenso no período estudado.

O coeficiente de escoamento superficial, calculado a partir das parcelas, foi muito baixo, representando menos de $1 \%$ do total precipitado em ambos os anos de estudo. A geração de escoamento superficial foi significativamente maior em 2009 em relação a 2008.

O potencial matricial da água do solo teve amplitude de 0 a $-85 \mathrm{kPa}$. Os maiores potenciais matriciais foram observados entre os meses de maio a agosto de 2008 , que coincidem com o período de menor precipitação. No entanto, o potencial matricial do solo variou, predominantemente, entre 0 a $-10 \mathrm{kPa}$, demonstrando que o solo permaneceu com alta umidade na maior parte do tempo no período estudado (Figura 6). O potencial matricial do ano de 2009 foi significativamente maior nas profundidades $0-0,15,0,15-0,30$ e $0,30-0,50 \mathrm{~m}$ que o ano 2008 , já na profundidade de 0,50-0,90 m não foram observadas diferenças significativas. 
Figura 6 - Precipitação semanal $(\mathrm{mm})$ e tensão da água no solo $(\mathrm{kPa})$. (a) porção mais baixa da vertente; (b) na porção central; (c) na parte superior.

Figure 6 - Weekly precipitation $(\mathrm{mm})$ and soil water tension $(\mathrm{kPa})$. (a) lower portion of the strand; (b) in the central portion; (c) at the top.

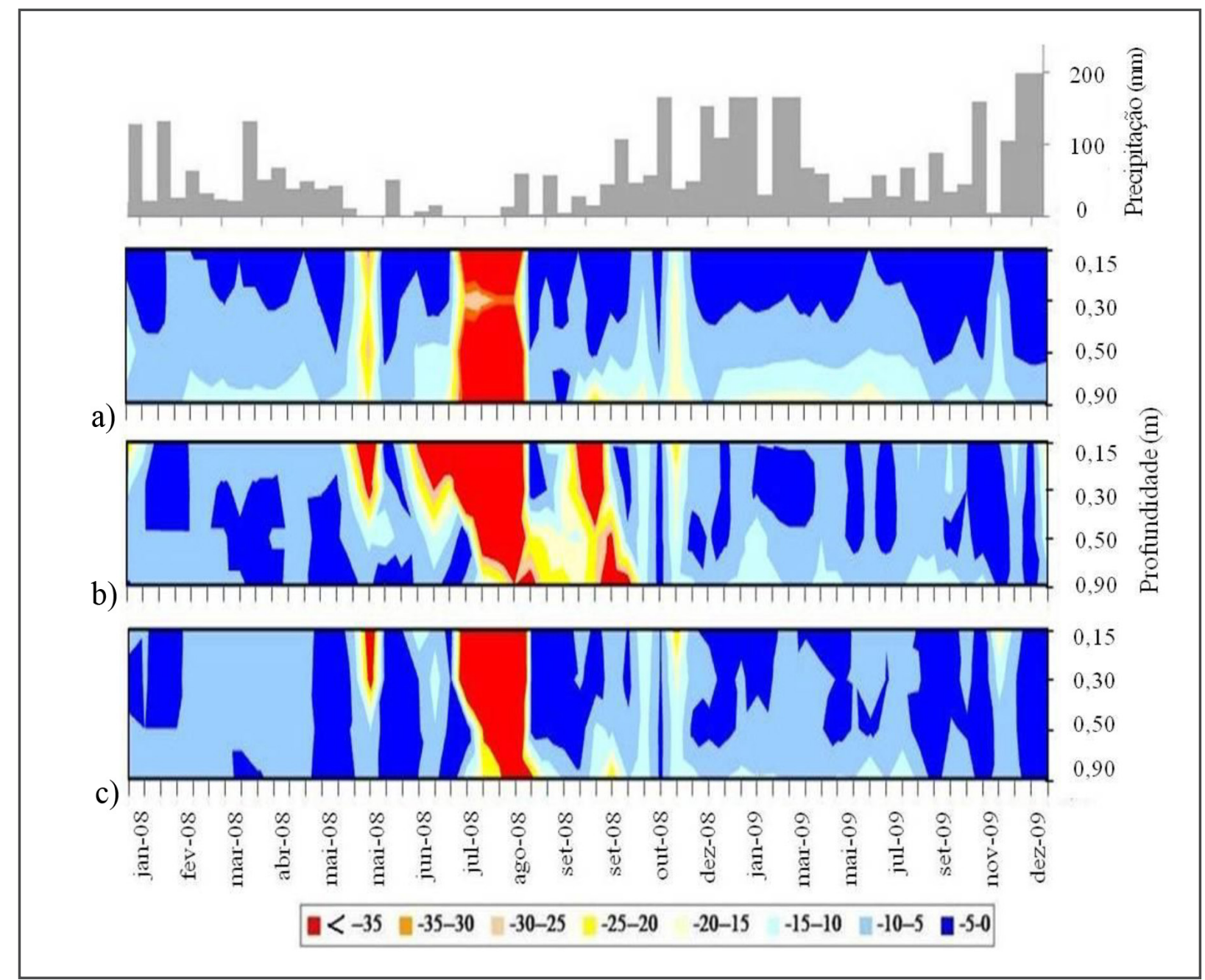

Fonte: Autores (2019)

O escoamento de base é a principal via hidrológica que compõe o deflúvio sendo representado por $78 \%$ e $75 \%$ do escoamento total nos anos de 2008 e 2009 , respectivamente.

O deflúvio e a razão deflúvio-precipitação $(\mathrm{Q} / \mathrm{P})$ foram respetivamente, $681 \mathrm{~mm}$ e de $40 \%$ em 2008, e 1.433 mm e a razão de $48 \%$ em 2009. O deflúvio diário do ano de 2009 foi significativamente maior que o de 2008.

A evapotranspiração calculada para o ano de 2008 foi de $1.035 \mathrm{~mm} \cdot \mathrm{ano}^{-1}\left(2,83 \mathrm{~mm} \cdot \mathrm{dia}^{-1}\right)$, sendo $461 \mathrm{~mm}$ pela transpiração e $574 \mathrm{~mm}$ pela evaporação, e em 2009 a evapotranspiração foi de $1.570 \mathrm{~mm} \cdot \mathrm{ano}^{-1}\left(4,3 \mathrm{~mm} \cdot \mathrm{dia}^{-1}\right)$, sendo $640 \mathrm{~mm}$ pela transpiração e $930 \mathrm{~mm}$ pela evaporação.

\section{Discussão}

Em relação à interceptação, a existência de um dossel de altura média de 15 metros com algumas árvores emergentes podendo alcançar até 30 metros, aliado a um alto índice de área foli- 
ar e abundância de epífitas (ALVES et al., 2010), acrescidas ao predomínio de chuvas de baixas intensidades são as prováveis razões da alta interceptação (32-33\%). Resultados similares foram observados em algumas florestas montanas que não sofrem influência significativa da neblina, que interceptam entre 20\% e 50\% da precipitação anual (EDWARDS, 1982; VIS, 1986; CAVELIER et al., 1997; BRUIJNZEEL; HAMILTON, 2000; GÓMEZ-PERALTA et al., 2008; BRAUMAN; FREYBERG; DAILY, 2010).

Segundo Bruijnzeel (1990), as florestas tropicais interceptam, em média, cerca de 13\% da precipitação anual. Fujieda et al. (1997) e Arcova, Cicco e Rocha (2003) apresentaram interceptação de 16,1\% e 18,6\%, em estudos também conduzidos no Parque Estadual da Serra do Mar, Núcleo de Cunha. Freitas et al. (2013) e Ávila et al. (2014) encontraram aproximadamente $20 \%$ do total precipitado, ambos em microbacias de Mata Atlântica no Estado de Minas Gerais, município de Viçosa e na Serra da Mantiqueira, respectivamente.

Como esperado, em função da diferença de pluviosidade anual e maiores intensidades de chuva, maiores precipitações internas ocorreram no ano de 2009 em relação ao ano de 2008.

Em relação $\mathrm{a}_{\text {sat }}$, os altos valores dessa variável observados próximo à superfície do solo podem ser atribuídos à vegetação densa que, pela presença de camada de serapilheira, atenua o impacto das gotas, evitando a formação de crostas no solo. Além disso, a deposição contínua de matéria orgânica na superfície favorece a atividade de organismos do solo que, por sua vez, favorecem a formação de macroporos (MARTINS, 2010).

A diminuição significativa nos valores de $\mathrm{K}_{\text {sat }}$ observada em relação às camadas mais profundas do solo podem causar consequências importantes nos processos hidrológicos, podendo gerar escoamento subsuperficial com o decréscimo acentuado de $\mathrm{K}_{\text {sat }}$ observado a 0,5 e 0,9 m (ELSENBEER et al., 1999). Apesar dos resultados dos potenciais matriciais durante a estação chuvosa, indicarem a possibilidade de formação de lençol suspenso, esse processo não foi observado no período estudado, considerando os resultados obtidos nos poços rasos $(0,5$ $\mathrm{m})$. Entretanto, algumas considerações podem ser feitas para explicar este fato: a) se ocorre escoamento subsuperficial, ele é de curta duração (GERMER et al., 2010) não podendo ser observado nas campanhas de campo; b) $\mathrm{K}_{\text {sat }}$ tem elevada variabilidade espacial e o lençol suspenso pode ocorrer apenas em alguns locais da bacia; c) $\mathrm{K}_{\text {sat }}$ pode ter sido subestimada, especialmente em profundidade, devido à escavação e compactação do solo pela utilização de trados (BAGARELLO, 1997; ZIMMERMANN; ELSENBEER; MORAES, 2006) e (d) o lençol suspenso pode estar localizado em profundidades menores ou maiores dos poços $(0,5 \mathrm{~m})$ construídos.

Assumindo a $\mathrm{K}_{\text {sat }}$ a 0,15 m de profundidade como representante das condições de infiltração da água no solo (MORAES et al., 2006), nota-se que o solo possui condições de acomodar a maior parte das intensidades de chuva. Em outras palavras, apenas $0,3 \%$ da intensidade de chuva da chuva excede a mediana de $\mathrm{K}_{\text {sat }}$ em tal profundidade.

Os valores de $\mathrm{K}_{\text {sat }}$ excedendo a maior parte das intensidades de chuva registradas podem explicar os altos valores de potencial matricial da água do solo um valor indireto da umidade do mesmo. De maneira geral, o solo permaneceu em condições muito úmidas quase todo período de estudo, essa constatação pode ser confirmada pelo baixo valor de escoamento superficial obtido no presente estudo.

Houve maior geração de escoamento superficial no ano 2009 em relação ao ano de 2008 atribuída à maior quantidade de água atingindo o solo via precipitação interna. Apesar de tal aumento, os valores de escoamento superficial continuam sendo relativamente baixos, o que colabora para justificar os potenciais matriciais da água do solo com valores altos, ou seja, refletindo alto conteúdo de umidade.

Com relação ao coeficiente de deflúvio diário, houve elevação significativa do deflúvio de 2008 para 2009. Essa observação pode ser atribuída à maior precipitação anual em 2009, aliada à maior quantidade de água que se infiltra, percola e escoa sobre o solo. Tais vias, em última análise, atingem os canais fluviais aumentando, desse modo, o deflúvio diário.

Acerca do deflúvio anual, um valor equivalente a 0,7 foi obtido no núcleo Cunha (FUJIEDA 
et al., 1997; DONATO et al., 2008). Esse valor é substancialmente maior que os do presente estudo (0,4 e 0,48 para, respectivamente, 2008 e 2009). Tais diferenças mostram que pode haver grande variabilidade espacial no tocante à dinâmica hidrológica em sub-bacias situadas dentro do bioma Mata Atlântica. Além disso, os resultados aqui apresentados evidenciam variabilidade temporal significativa causada por diferentes pluviosidades anuais sobre vários processos hidrológicos de uma mesma bacia dentro do referido bioma.

\section{Conclusões}

Com os resultados obtidos no presente trabalho, pode-se concluir que a maior precipitação apresentada em 2009 em relação a 2008 causou aumentos significativos na geração da precipitação interna, escoamento superficial e deflúvio diário. Além disso, devido à alta condutividade hidráulica saturada e baixa intensidade da chuva, o escoamento superficial mostrou-se baixo em relação à chuva anual, apenas $1 \%$ do total precipitado.

Cabe salientar que mais estudos como esse são necessários para compreender como as alterações climáticas afetam os processos hidrológicos. Em especial, anos de baixa pluviosidade, por sua criticidade, devem ser examinados uma vez que tem implicações relevantes para na perspectiva de gerenciamento de gerenciar os recursos hídricos.

\section{Agradecimentos}

À Fundação de Amparo à Pesquisa no Estado de São Paulo - FAPESP, pela bolsa de estudo concedida n. 06/51488-0 e pelo projeto de pesquisa n. 06/55136-0. Ao Centro de Energia Nuclear na Agricultura (CENA/USP) e a Escola Superior de Agricultura (ESALQ/USP), por fornecer os meios necessários para a realização desse trabalho.

\section{Referências}

ALVES, L. F. et al. Forest structure and live above ground biomass variation along an elevation gradient of tropical Atlantic moist forest (Brazil). Forest Ecology and Management, Amsterdam, v. 260 , n. 6, p. 679-691, jul. 2010.

AMOOZEGAR, A. Compact constant head permeameter: a convenient device for measuring hydraulic conductivity. In: TOPP, C. G. (ed.). Advances in measurement of soil physical properties: bringing theory into practice. Madison: SSSA, 1992. p. 31-42.

ARCOVA, F. C. S.; CICCO, V.; ROCHA, P. A. B. Precipitação efetiva e interceptação das chuvas por floresta de Mata Atlântica em uma microbacia experimental em Cunha - São Paulo. Revista Árvore, Viçosa, MG, v. 27, p. 257-262, mar./abr. 2003.

ARCOVA, F. C. S.; CICCO, V. Características do deflúvio de duas microbacias hidrográficas no Laboratório de Hidrologia Florestal Walter Emmerich, Cunha - SP. Revista Instituto Florestal, São Paulo, v. 9, n. 2, p. 153-170, jun. 1997.

ÁVILA, L. F. et al. Participação da precipitação pluvial em um microbacia hidrográfica ocupada por mata atlântica na Serra da Mantiqueira. Ciência Florestal, Santa Maria, v. 24, n. 3, p. 583595, jul./set. 2014.

BAGARELLO, V. Influence of well preparation on field saturated hydraulic conductivity measured with the Guelph Permeameter. Geoderma, Amsterdam, v. 80, n. 1/2, p. 169-180, out. 1997.

BRAUMAN, K. A.; FREYBERG, D. L.; DAILY, G. C. Forest structure influences on rainfall partitioning and cloud interception: a comparison of native forest sites in Kona, Hawai'i. Agricultural and Forest Meteorology, New York, v. 150, n. 2, p. 265-275, fev. 2010. 
BRUIJNZEEL, L. A. Hydrology of moist tropical forests and effects of conversion: a state of knowledge review. Paris: UNESCO; IHP, 1990. 224 p.

BRUijnzeEl, L. A.; HAMilton, L. S. Decision Time For Cloud Forests, Paris, v. 13, n. 7, p. 44, 2000.

CAVELIER, J. et al. Water balance and nutrient inputs in bulk precipitation in tropical montane cloud forest in Panama. Journal of Hydrology, Amsterdam, v. 193, n. 1/4, p. 83-96, jun. 1997.

CHOW, V. T.; MAIDMENT, D. R.; MAYS, L. W. Applied hydrology. New York: McGraw-Hill, 1988. 570 p.

DONATO, C. F. et al. Balanço de massa em microbacia experimental com Mata Atlântica, na Serra do Mar, Cunha-SP. Revista Instituto Florestal, São Paulo, v. 20, n. 1, p. 1-11, jun. 2008.

EDWARDS, P. J. Studies of mineral cycling in a montane rain forest in New Guinea, V. Rates of cycling in throughfall and litter fall. Journal of Ecology, Oxford, v. 70, p. 807-827, 1982.

ELSENBEER, H. Hydrologic flowpaths in tropical rainforest soilscapes - a Review. Hydrological Process, Nova Jersey, v. 15, n. 4, p. 1751-1759, 2001.

ELSENBEER, H. M. et al. Hydraulic conductivities of latosols under pasture, forest and teak in Rondônia, Brazil. Hydrological Processes, Nova Jersey, v. 13, n. 9, p. 1417-1422, jun. 1999.

FILL, H. D. Informações hidrológicas. In: BARTH, F. T. et al. Modelos para gerenciamento de recursos hídricos. São Paulo: Nobel,1987. cap. 2, p. 95-210.

FREITAS, J. P. O. et al. Distribuição da água de chuva em Mata Atlântica. Revista Ambiente \& Água, Taubaté, v. 8, n. 2, p. 100-108, abr. 2013.

FUJIEDA, M. et al. Hydrological processes at two subtropical forest catchments: the Serra do Mar, São Paulo, Brazil. Journal of Hydrology, Amsterdam, v. 196, n. 1/4, p. 26-46, set. 1997.

FUNDAÇÃO SOS MATA ATLÂNTICA. Atlas dos remanescentes florestais da Mata Atlântica Período 2000-2005. São Paulo: INPE, 2006.

GERMER, S. et al. Influence of land-use change on near-surface hydrological processes: undisturbed forest to pasture. Journal of Hydrology, Amsterdam, v. 380, n. 3/4, p. 473-480, jan. 2010.

GÓMEZ-PERALTA, D. et al. Rainfall and cloud-water interception in tropical montane forests in the eastern Andes of Central Peru. Forest Ecology and Management, Amsterdam, v. 255, n. 3/4, p. 1315-1325, mar. 2008.

GROPPO, J. D. Caracterização hidrológica e dinâmica do nitrogênio em uma microbacia com cobertura florestal (Mata Atlântica), no Parque Estadual da Serra do Mar, Núcleo Santa Virgínia. 2010. Tese (Doutorado em Ciências) - Centro de Energia Nuclear na Agricultura, Universidade de São Paulo, Piracicaba, 2010.

JIPP, P. H. et al. Deep soil moisture storage and transpiration in forests and pastures of seasonallydry Amazonia. In: MARKHAM, A. (ed.). Potential impacts of climate change on tropical forest ecosystems. Dordrecht: Springer, 1998. v. 39, p. 395-412.

LEITÃO-FILHO, H. F. Considerações sobre florística de florestas tropicais e subtropicais do Brasil. IPEF, [s. l.], v. 35, p. 41-46, abr. 1987.

LIRA, P. K. et al. Land-use and land-cover change in the Atlantic forest landscapes. Forest Ecology and Management, Amsterdam, v. 278, p. 80-89, ago. 2012.

MARTINS, S. C. Caracterização dos solos e serapilheira ao longo do gradiente altitudinal da Mata Atlântica, estado de São Paulo. 2010. Tese (Doutorado em Ciências) - Centro de Energia Nuclear na Agricultura, Universidade de São Paulo, Piracicaba, 2010. 
MORAES, J. M. et al. Water storage and runoff processes in plinthic soils under forest and pasture in Eastern Amazonia. Hydrological Processes, Chichester, v. 20, n. 12, p. 2509-2526, jul. 2006.

MYERS, N. et al. Biodiversity hotspots for conservation priorities. Nature, London, v. 403, p. 852-858, fev. 2000.

MURRAY-SMITH, C. et al. Plant diversity hotspots in the Atlantic coastal forest of Brazil. Conservation Biology, Nova Jersey, v. 23, n. 1, p. 151-163, out. 2009.

RIBEIRO, M. C. et al. The Brazilian Atlantic Forest: How much is left, and how is the remaining forest distributed? Implications for conservation. Biological Conservation, Amsterdam, v. 142, n. 6, p. 1141-1153, jun. 2009.

ROCHA, H. R. et al. Seasonality of water and heat fluxes over a tropical Forest in eastern Amazonia. Ecological Applications, Washington, v. 14, n. 4, p. 22-32, ago. 2004.

SÃO PAULO. Secretaria do Meio Ambiente. Instituto Florestal. Parque Estadual da Serra do Mar - Plano de Manejo. São Paulo: Secretaria do Meio Ambiente; Instituto Florestal, 2006.

TABARELLI, M.; MANTOVANI, W. A regeneração de uma floresta tropical montana após corte e queima (São Paulo - Brasil). Revista Brasileira de Biologia, São Carlos, v. 59, n. 2, p. 239-250, 1999.

TUCCI, C. E. M. Hidrologia: ciência e aplicação. 1. ed. Porto Alegre: ABRH, 2001. 943 p. (Coleção ABRH Recursos Hídricos, 4).

VELOSO, H. P.; RANGEL FILHO, A. L. R.; LIMA, J. C. A. Classificação da vegetação brasileira, adaptada a um sistema universal. Rio de Janeiro: IBGE, Departamento de Recursos Naturais e Estudos Ambientais, 1991.

VIS, M. Interception, drop size distributions and rainfall kinetic energy in four Colombian forest ecosystems. Earth Surface Process and Landforms, Reino Unido, v. 11, n. 6, p. 591-603, nov./ dez. 1986.

ZIMMERMANN, B.; ELSENBEER, H.; MORAES, J. M. The influence of land-use changes on soil hydraulic properties: implications for runoff generation. Forest Ecology and Management, Amsterdam, v. 222, n. 1/3, p. 29-38, fev. 2006. 